\title{
FEM Simulations and Optimization about Residual Stresses in Coating Structures with Functionally Graded Materials Layer
}

\author{
Chenguang Huang, Siying Chen and Zhuping Duan \\ State Key Laboratory of Nonlinear Mechanics, Institute of Mechanics, Chinese Academy of Sciences, \\ Beijing 100080, P.R. China
}

Keywords: Finite Element Method, Optimization, Thermal spraying

\begin{abstract}
An elasto-plastic finite element method is developed to predict the residual stresses of thermal spraying coatings with functionally graded material layer. In numerical simulations, temperature sensitivity of various material constants is included and mixture law is used to depict the constitutive behaviors of FGM layer. The optimized distribution form of the volume fractions of constituents in FGM is obtained by the first order optimization method in the $\mathrm{AL}_{2} \mathrm{O}_{3}-\mathrm{Ni}$ system. At the same time, Effects of geometry and materials behaviors on the optimization result are also investigated numerically. When the length of specimen, the width of FGM layer and thermal expanding coefficient of the substrate material increase, the optimized distribution parameter $p$ decreases obviously. It is found that the optimization of the constituent contents in FGM reduces the magnitude of residual stresses to a large degree and makes the maximum residual stresses to shun the weakest part of the coating structure.
\end{abstract}

\section{Introduction}

Thermal spraying coatings have been used widely in many fields such as aviation industry, power engineering, because this kind of materials system possesses synchronously the perfect properties against the high temperature, wearing and eroding of ceramic materials, and high toughness of substrate materials [1].

In coating structures, the apparent differences of thermal physical and mechanical properties exist between the substrate material and ceramic coating. The mismatch will result in the concentration of stresses and the deformation in the interface between the substrate and the ceramic layer, when the coating structure is cooled in the production process. Specially, in the intersection region of interface and free edge, the singularity of stresses will occur and induce the initiation of micro crack. Hsueh et al [2-7] investigated the dependences of magnitude of residual stresses on the sample dimension, materials properties etc. by using the experimental observations, analytical solutions and numerical simulation method respectively.

In order to improve the service performance of thermal spraying coatings, the concept of functionally graded materials (FGM) was introduced. People believe that the FGM layer between the substrate and coating layer can decrease the residual stress in the materials system to some degree, at the same time, and can upgrade the interface strength. However, how to select the appropriate microstructure and distribution forms of various constituent contents in FGM layer, which agitates the new interesting of research work. Some important conclusions were obtained and presented by Kawasaki et al [8-11] and Drake and his colleagues [12-13] in their papers respectively. But, they did not use the iteration optimization algorithm, and only discussed several special cases of the designing variables space.

In this paper, some elementary knowledge about the optimization is introduced and applied in the FEM code. The plastic deformations of FGM layer and the substrate material, and the dependence of material properties on the temperature are considered in the numerical simulations, to obtain an accurate description about the mechanical responses of thermal spraying coatings. 


\section{Problem Formulation}

In Fig. 1, the typical coating structure with FGM layer is shown. The $\mathrm{Al}_{2} \mathrm{O}_{3}-\mathrm{Ni}$ is selected as the subject investigated. $\mathrm{Al}_{2} \mathrm{O}_{3}$ is used as the ceramic coating against high temperature, while the $\mathrm{Ni}$, a kind of refractory alloy, is adopted as substrate materials. In this paper, an assumption temperature dropping from $800 \mathrm{~K}$ to $300 \mathrm{~K}$ is used to study the residual stresses resulted from the cooling in the production process.

In the two-dimensional plane strain model, the controlling equations and the boundary conditions are,

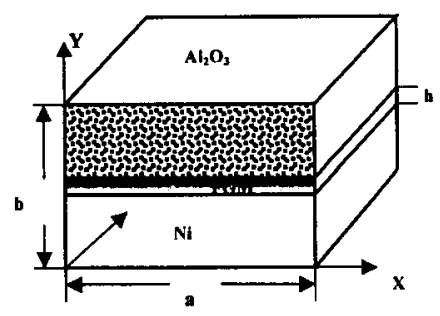

Fig. 1 Sketch map of coating structures with FGM layer

$$
\begin{aligned}
& \sigma_{i, j}=0 . \\
& \varepsilon=\varepsilon^{e}+\varepsilon^{p}+\varepsilon^{\theta}=\frac{1}{2}\left(u_{i, j}+u_{j, i}+u_{i, k} u_{j, k}\right) . \\
& \sigma_{i j}=\lambda \varepsilon_{k k}{ }^{e} \delta_{i j}+2 \mu \varepsilon_{i j}^{e}, \varepsilon_{i j}^{\theta}=\alpha \Delta T \delta_{i j} \Delta T=-500 K . \\
& \sigma_{i !}=0, \quad x=a \text { or } y=0, b . \\
& u_{1}=0, \sigma_{12}=0, \quad x=0 . \\
& \sigma_{i j}=u_{i}=0, \text { at } T=800 \mathrm{~K} .
\end{aligned}
$$

where, $u_{\mathrm{i}}, \sigma_{\mathrm{ij}}, \varepsilon_{\mathrm{ij}}, \varepsilon_{\mathrm{ij}}^{\mathrm{e}}, \varepsilon_{\mathrm{ij}}^{\mathrm{p}}$, and $\varepsilon_{\mathrm{ij}}{ }^{\theta}$ denote the displacement, stress, strain, elastic strain, plastic strain and temperature strain, $\lambda$ and $\mu$ are the elastic constants, $\alpha$ and $\Delta T$ are the thermal expending coefficient and temperature rising respectively.

The simple linear elastic model is used to describe the behaviors of $\mathrm{Al}_{2} \mathrm{O}_{3}$, while isotropic hardening bilinear model is considered as constitutive law of alloy substrate. As to the FGM layer, the mixture law, which was presented by Tamura et al [14] and shown in Fig. 2, is applied in this paper,

$$
\begin{aligned}
& \sigma_{c}=V_{\alpha} \sigma_{\alpha}+V_{\beta} \sigma_{\beta} . \\
& \varepsilon_{c}=V_{\alpha} \varepsilon_{\alpha}+V_{\beta} \varepsilon_{\beta} . \\
& V_{\alpha}+V_{\beta}=1, \quad q=\left|\frac{\sigma_{\alpha}-\sigma_{\beta}}{\varepsilon_{\alpha}-\varepsilon_{\beta}}\right| .
\end{aligned}
$$

where $q=4.5 \mathrm{GPa}, \sigma_{\alpha}, \sigma_{\beta}$ and $\sigma_{\mathrm{c}}$ denote the true stresses in ceramic phase, alloy and graded layer, $\varepsilon_{\alpha}$, $\varepsilon_{\beta}$ and $\varepsilon_{\mathrm{c}}$ are the corresponding strains, $V_{\alpha}$ and $V_{\beta}$ denote the volume contents of two constituents in FGM.

Tab. 1 Materials parameters of $\mathrm{Al}_{2} \mathrm{O}_{3}$ and $\mathrm{Ni}$

\begin{tabular}{ccccccc}
\hline Material & $\begin{array}{c}\text { Temperature } \\
{[\mathrm{K}]}\end{array}$ & $\begin{array}{c}\text { Yield } \\
\text { stress } \\
{[\mathrm{MPa}]}\end{array}$ & $\begin{array}{c}\text { TEC } \\
{\left[\mathrm{K}^{-1}\right]}\end{array}$ & $\begin{array}{c}\text { Youngs } \\
\text { Modulus [GPa] }\end{array}$ & $\begin{array}{c}\text { Hardening modulus } \\
{[\mathrm{GPa}]}\end{array}$ & $\begin{array}{c}\text { Poisson } \\
\text { rate }\end{array}$ \\
\hline $\mathrm{Al}_{2} \mathrm{O}_{3}$ & $/$ & $/$ & $7.4 \mathrm{e}-6$ & 380 & $/$ & 0.25 \\
\hline \multirow{5}{*}{ Nickel } & 300 & 148 & $10.4 \mathrm{e}-6$ & 74 & 0.67 & 0.31 \\
& 400 & 153 & $11.4 \mathrm{e}-6$ & 76 & 0.66 & 0.31 \\
& 500 & 140 & $12.4 \mathrm{e}-6$ & 70 & 0.65 & 0.31 \\
& 600 & 138 & $13.4 \mathrm{e}-6$ & 69 & 0.64 & 0.31 \\
& 700 & 115 & $14.4 \mathrm{e}-6$ & 57 & 0.33 & 0.31 \\
& 800 & 100 & $15.4 \mathrm{e}-6$ & 53 & 0.22 & 0.31 \\
\hline
\end{tabular}




$$
V_{\alpha}=\left(y^{\prime} / h\right)^{n} \quad V_{\beta}=1-V_{\alpha}
$$

where $h$ is the width of FGM layer, $y^{\prime}$ denotes the distance to the upper surface of the substrate. $p$ is the controlling factor of content distributions of various constituents, and is selected as optimization design variables (ODV).

In Table1, the mechanical parameters of ceramic coating material and the substrate are listed.

\section{Optimization Algorithm}

In this section, we introduce the first order optimization method simply, which is used in our work. The optimization design variables can be denoted as following vector [15],

$$
\begin{aligned}
& \tilde{x}=\left[\begin{array}{llll}
x_{1} & x_{2} & \cdots & x_{n}
\end{array}\right] . \\
& x_{i}^{\prime} \leq x_{i} \leq x_{i}^{\prime \prime}, \quad(i=1,2,3, \ldots n) .
\end{aligned}
$$

where Eq.13 describes the span of design variables. $n$ is the number of design variables. The objective function is indicated by,

$$
f=f(\tilde{x}) \text {. }
$$

In the optimization, the constraint conditions can be expressed by the status variables, which are the functions of design variables.

$$
\begin{aligned}
& \begin{cases}g_{i}(\widetilde{x}) \leq g_{i}^{\prime \prime} & \left(i=1,2,3, \ldots m_{1}\right) \\
h_{i}^{\prime} \leq h_{i}(\widetilde{x}) & \left(i=1,2,3, \ldots m_{2}\right) \\
w_{i}^{\prime} \leq w_{i}(\widetilde{x}) \leq w_{i}^{\prime \prime} & \left(i=1,2,3, \ldots m_{3}\right)\end{cases} \\
& m=m_{1}+m_{2}+m_{3} .
\end{aligned}
$$

Where $g_{\mathrm{i}}, h_{\mathrm{i}}$ and $w_{\mathrm{i}}$ are the status variables, $m$ is the total number of various kind of constraint conditions. By now, a complete mathematic representation about optimization problem is accomplished.

The unconstrained, non-dimensional objective function, is written as,

$$
Q(\tilde{x}, q)=\frac{f}{f_{0}}+\sum_{i=1}^{n} p_{x}(\widetilde{x})+q\left[\sum_{i=1}^{m_{1}} p_{g}\left(g_{i}\right)+\sum_{i=1}^{m_{2}} p_{h}\left(h_{i}\right)+\sum_{i=1}^{m_{1}} p_{w}\left(w_{i}\right)\right]
$$

where $f_{0}$ is the reference objective function value, $q$ is the response surface parameter, $p_{\mathrm{x}}, p_{\mathrm{y}}, p_{\mathrm{h}}$ and $p_{\mathrm{w}}$ are the penalties. The design variables are as followings, in the iteration step of $j+1$,

$$
\begin{aligned}
& \widetilde{x}^{(j+1)}=\widetilde{x}^{(j)}+s_{,} \widetilde{d}^{(j)} \\
& 0 \leq s_{,} \leq \frac{s_{\max }}{100} s^{*}, .
\end{aligned}
$$

where, $\tilde{d}^{(j)}$ is the search direction vector, $s_{\mathrm{j}}$ is the linear search step size. $S_{\mathrm{j}}^{*}$ and $s_{\max }$ are the maximum possible step size and coefficient. $\tilde{d}^{(j)}$ can be obtained by the Polak-Ribiene method,

$$
\begin{aligned}
& j=0, \quad \tilde{d}^{(0)}=-\widetilde{\nabla} Q\left(\widetilde{x}^{(0)}, q\right)=\widetilde{d}_{f}^{(0)}+\tilde{d}_{p}^{(0)}=-\widetilde{\nabla} Q_{f}\left(\widetilde{x}^{(0)}\right)-\widetilde{\nabla} Q_{p}\left(\widetilde{x}^{(0)}\right) . \\
& j \geq 1, \quad \widetilde{d}^{(j)}=-\tilde{\nabla} Q\left(\widetilde{x}^{(j)}, q_{k}\right)+r_{j-1} \widetilde{d}^{(j-1)} . \\
& r_{j-1}=\frac{\left[\widetilde{\nabla} Q\left(\widetilde{x}^{(j)}, q\right)-\tilde{\nabla} Q\left(\tilde{x}^{(j-1)}, q\right)\right]^{7} \tilde{\nabla} Q\left(\widetilde{x}^{(j)}, q\right)}{\left|\widetilde{\nabla} Q\left(\widetilde{x}^{(j-1)}, q\right)\right|^{2}} .
\end{aligned}
$$




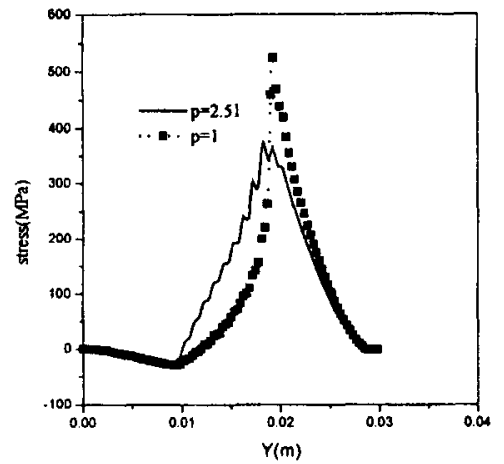

Fig. 3 Normal stresses of y-direction in free edge

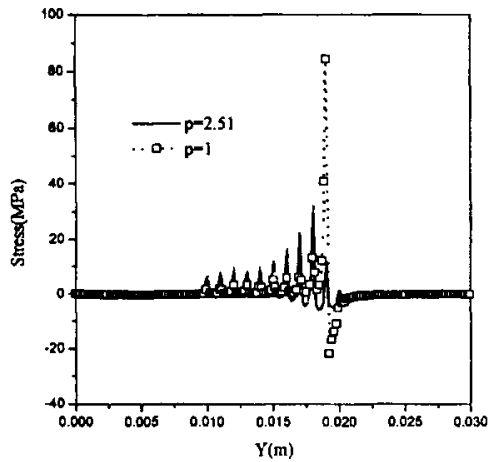

Fig. 4 Shear stresses in free edge

$$
\frac{\partial Q\left(\widetilde{x}^{(\prime)}\right)}{\partial x_{i}} \approx \frac{Q\left(\widetilde{x}^{(\prime)}+\Delta x_{i} \widetilde{e}\right)-Q\left(\tilde{x}^{(\prime)}\right)}{\Delta x_{i}} .
$$

Convergence is assumed when Eq.24 is satisfied,

$$
\left|f^{(1)}-f^{(1-1)}\right| \leq \tau \text { and }\left|f^{(i)}-f^{(b)}\right| \leq \tau \text {. }
$$

where $f^{(b)}$ is the optimized objective function value. In this paper, $p$ is considered as a design variable.

\section{FEM Simulation Results and Discussions}

The stresses and deformation in the thermal spraying coating structure, which is shown in Fig.1, are investigated by finite element method with the introduction of optimization algorithm. The constitutive law and various materials properties are given in the Section 2 .

Calculation results show that the optimized $p$ is 2.51 at $a=30 \mathrm{~mm}, b=30 \mathrm{~mm}, h=10 \mathrm{~mm}$. In Figs. 3 and $4, \sigma_{\mathrm{y}}$ and $\tau_{\mathrm{xy}}$ and their distributions along free edge of $x=a$ are shown with the optimized $p$ value (2.51) and $p=1$, which means linear distributions of contents of constituent materials.

It was reported that the introduction of the FGM layer could reduce obviously the residual stresses induced by the mismatch of materials properties in the coating structure. From Fig. 3 and 4, it is proved that optimization of content distribution parameter $p$, will decrease further the residual stresses in the materials system. In our calculation, the maximum value of $\sigma_{\mathrm{y}}$ drops about $30 \%$ and $\sigma_{\mathrm{xy}}$ in the interface decreases over $50 \%$ when $p$ is changed from 1 to optimized value 2.51 . However, it is these stress components that play the most important roles in the spalling and debonding failure of coatings.

Fig. 5 shows distributions of $\sigma_{\mathrm{y}}$ and $\tau_{\mathrm{xy}}$ in the whole region investigated, when $p=1$ or 2.51 respectively. A significant and interesting phenomenon is also presented in this figure: through the optimization of constituents contents distributions, the residual stresses in coating structures can be reduced obviously, at the same time, the position, where the maximum residual stresses occur, is moved from the interface and free edge to the inner of FGM layer. It means that the optimization designing makes the most dangerous stress condition to deviate from the most vulnerable region.

It is known that the changes of dimension, material parameters of coating structures will alter the amplitude and distributions of residual stresses. In this paper, the effects of geometry and material properties are studied by numerical simulations, and shown in Fig. 6-8.

Fig. 6 shows the dependence of optimized $p$ value on the ratio between the length and width of samples. In simulations, the widths of samples and FGM layer are fixed in $30 \mathrm{~mm}$ and $10 \mathrm{~mm}$, the length of samples is changed in the region of $10 \mathrm{~mm}$ to $50 \mathrm{~mm}$. It is found that the optimized $p$ decreases with the enlargement of samples length.

However, when the length and width of samples are $10 \mathrm{~mm}, 30 \mathrm{~mm}$, and kept as constants, the 
optimized variable $p$ decreases from 6.01 to 3.40 with the width of FGM layer increases from $2 \mathrm{~mm}$ to $10 \mathrm{~mm}$. From the Fig. 6 and 7, a conclusion can be made that the content distributions of constituent materials tend toward the linear form, $p=1$, with the continuous increments of samples length or width of FGM layer.

In the above FEM calculations and optimization process, the materials constants listed in Section 2 are adopted. Now thermal expanding coefficient of substrate materials is varied artificially in order to research its effect to the optimized variable. The length of samples $a=10 \mathrm{~mm}$, the widths of samples and FGM layer are $30 \mathrm{~mm}$ and $5 \mathrm{~mm}$. We find that the optimized $p$ value increases with the reduction of TEC of the substrate material, from $15.4 \times 10^{-6} \mathrm{~K}^{-1}$ to $5 \times 10^{-6} \mathrm{~K}^{-1}$, as shown in Fig. 8 .

In the optimization process, the selection of objective function is very important. We used some different objective functions in this work, such as $\sigma_{y}, \tau_{\mathrm{xy}}, \sigma^{\text {eff }}$ and $\varepsilon_{\mathrm{p}}^{\text {eff }}$. At last, a pure empirical conclusion is obtained, that $\sigma_{\mathrm{y}}$ is the most adequate objective function. When other objective functions are adopted, the optimization process will attend to one thing and lose another.

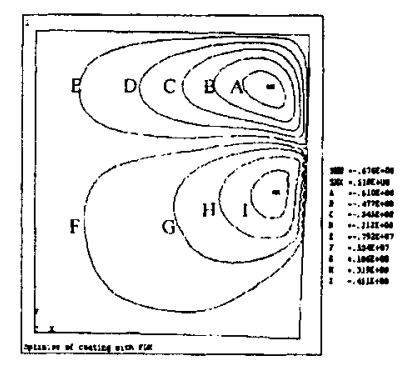

(a) $\tau_{\mathrm{xy}}, p=2.51$

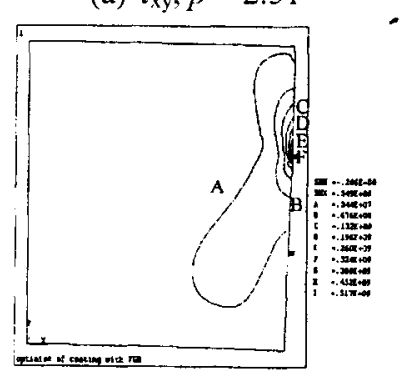

(d) $\sigma_{y}, p=1$

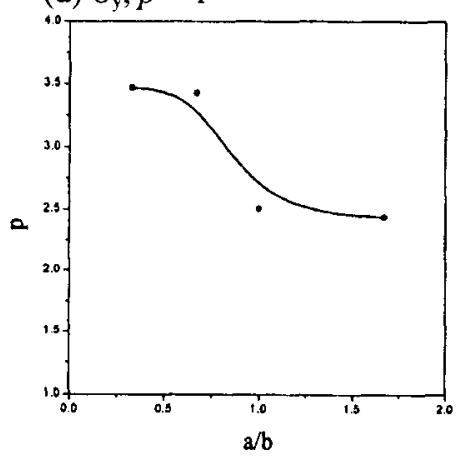

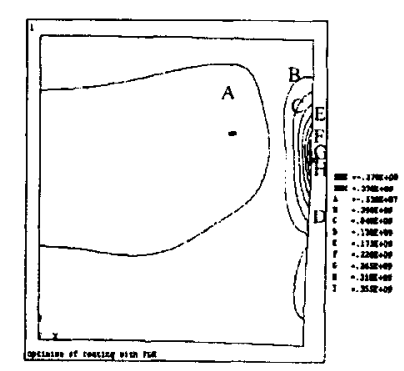

(b) $\sigma_{y}, p=2.51$

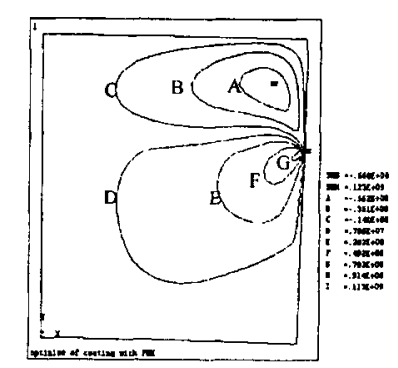

(c) $\tau_{x y}, p=1$

Fig. 5 Distribution of $\sigma_{y}$ and $\tau_{x y}$ before and after the optimization analysis

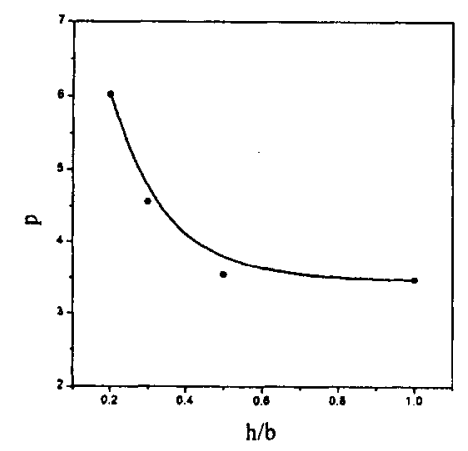

Fig. 6 Effect of ratio between length and width of Fig. 7 Dependence of optimized $p$ on the width of samples on the optimized $p$

FGM layer 


\section{Concluding Remarks}

In this paper, the finite element method and optimization theory are applied to investigate residual stresses in thermal spraying coatings with FGM layer. The main results are as followings,

(1) The optimization of volume content distributions of constituent materials can reduce the residual stress to a large degree, compared with one of linear distribution, and can make the residual stresses peak values depart from the weak region of coating structures.

(2) Effects of samples dimension and thermal expanding coefficient of the substrate material on

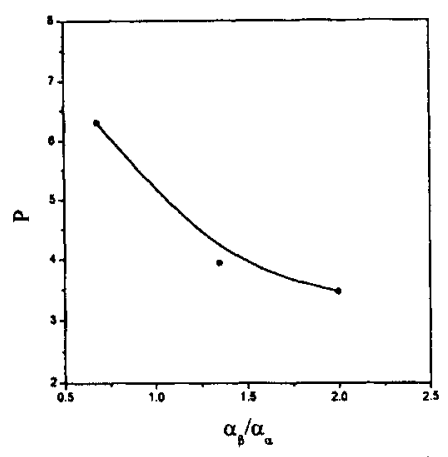

Fig. 8 Relation between the optimized $p$ value and TEC of the substrate material the optimized variable $p$ are investigated in detail. The optimized $p$ will decrease with increments of width of FGM layer, ratio between the length and width of samples and the TEC of the substrate material.

In the future work, the similar research will be done for the optimization of thermal stresses of coatings, when the multi-layer structure is used as a hot-end component. In addition, the effect of bonding layer will be probed detailedly.

\section{Acknowledgements}

This work is supported by the National Natural Science Foundation of China (NSFC), with contract numbers of 19891180 and 10002021.

\section{References}

[1] V. V. Sobolev, J. M. Guilemany, J. Nutting, et al: Int. Mater. Review Vol. 42(1997), pp.117.

[2] C. H. Hsueh, A. G. Evans: J. Am. Ceram. Soc. Vol. 68(1985), pp.241.

[3] E. Reimanis, B. J. Dalgleish, A. G. Evans: Acta. Metall. Mater. Vol. 39(1987), pp.110.

[4] V. V. Lyubimov, A. A. Voevodin, S. E. Spassky, et al: Thin Solid Films Vol. 207(1992), pp. 117.

[5] C. C. Chiu: Sci. Eng. A Vol. 150(1992), pp.139.

[6] M. Hu: J. Appl. Phys. Vol. 70(1991), pp. R53.

[7] W. A. Zdaniewski, J. C. Conway, H. P. Kirchner: J. Am. Ceram. Soc. Vol. 70(1987), pp. 110.

[8] A. Kawasaki, R. Watanabe: J. Jap. Inst. Metals Vol. 51(1987), pp.51.

[9] A. Kawasaki, R. Watanabe: Proceedings of the international institute for science of sintering (IISS) symposium, (Elsevier Science, England, 1988), pp.1197.

[10]T. Hirai, M. Sasaki: JSME Int. J. Vol. 34(1991), pp.123.

[11] T. Hirano, T. Yamada, J. Teraki, et al: Proceedings of the $16^{\text {th }}$ international symposium on space technology and science, (AGEN, Japan, 1988), pp.375.

[12]R. L. Williamson, B. H. Rabin, J. T. Drake: J. Appl. Phys. Vol. 74(1993), pp.1310.

[13]J. T. Drake, R. L. Williamson, B. H. Rabin: J. Appl. Phys, Vol. 74(1993), pp.1321.

[14]T. Tamura, Y. Tomota, H. Ozawa: Proceedings of the third international conference on strength of metals and alloys. (Institute of Metal and Iron and Steel Institute, England, 1973), pp.611.

[15]ANSYS Co.: ANSYS theoretical Manual (Electronic Press, 1997). 


\section{Functionally Graded Materials VII}

10.4028/www.scientific.net/MSF.423-425

FEM Simulations and Optimization about Residual Stresses in Coating Structures with Functionally Graded Materials Layer

10.4028/www.scientific.net/MSF.423-425.659 